\title{
Distant influences of amygdala lesion on visual cortical activation during emotional face processing
}

\author{
Patrik Vuilleumier ${ }^{1,2}$, Mark P Richardson ${ }^{3}$, Jorge L Armony ${ }^{2,4}$, Jon Driver ${ }^{2,5}$ \& Raymond J Dolan ${ }^{2,5}$ \\ Emotional visual stimuli evoke enhanced responses in the visual cortex. To test whether this reflects modulatory influences \\ from the amygdala on sensory processing, we used event-related functional magnetic resonance imaging ( $\mathrm{fMRI}$ ) in human \\ patients with medial temporal lobe sclerosis. Twenty-six patients with lesions in the amygdala, the hippocampus or both, \\ plus 13 matched healthy controls, were shown pictures of fearful or neutral faces in task-releant or task-irrelevant positions \\ on the display. All subjects showed increased fusiform cortex activation when the faces were in task-relevant positions. Both \\ healthy individuals and those with hippocampal damage showed increased activation in the fusiform and occipital cortex \\ when they were shown fearful faces, but this was not the case for individuals with damage to the amygdala, even though \\ visual areas were structurally intact. The distant influence of the amygdala was also evidenced by the parametric \\ relationship between amygdala damage and the level of emotional activation in the fusiform cortex. Our data show that \\ combining the $\mathrm{fMRI}$ and lesion approaches can help reveal the source of functional modulatory influences between distant \\ but interconnected brain regions.
}

\begin{abstract}
Emotion can affect perception, memory and behavior ${ }^{1,2}$, with emotional events receiving prioritized attention and enhanced processing $^{3-6}$. Functional neuroimaging studies show that emotionally negative (for example, fear-related) visual stimuli activate not only brain areas traditionally implicated in emotion, such as the amygdala $^{1,7}$, but also the visual cortex ${ }^{8-10}$. Thus, seeing faces with fearful expressions produces greater activation of the face-responsive areas of the fusiform cortex than does seeing faces with neutral expressions $^{9,11,12}$. Similarly, face-selective neurons in the monkey temporal cortex show enhanced responses to threatening expressions as compared to neutral expressions ${ }^{13}$. It has been speculated that such increases in neuronal activity may reflect a boost in perceptual analysis caused by "feedback" or reentrant influences from the amygdala ${ }^{14-16}$. Here we provide a direct test of this proposal using functional neuroimaging in human subjects with or without amygdala damage.

The amygdala has a central role in fear processing ${ }^{1,17-19}$. It has extensive connections with many cortical and subcortical areas, including back-projections to the visual cortex that may modulate sensory processing in these areas based on emotional signals ${ }^{20,21}$. To date, there was only indirect evidence supporting this putative modulatory role ${ }^{9,22}$. Monkeys with bilateral amygdala lesions disregard aversive visual stimuli ${ }^{18,23}$, and in humans, surgical excision of temporal regions, including the amygdala, can abolish the detection advantage for negative over neutral stimuli ${ }^{4}$. But no study has directly tested whether amygdala damage specifically disrupts the enhanced neural response to emotional stimuli in distant visual areas.
\end{abstract}

Using whole-brain fMRI in humans, we tested the prediction that lesions in the amygdala would eliminate the enhanced activation typically exhibited by the occipital and fusiform visual areas in response to fearful (compared to neutral) faces, even when these areas remained intact. We studied patients with the well-characterized syndrome of medial temporal lobe sclerosis ${ }^{24,25}$, either involving or sparing the amygdala ${ }^{26,27}$. These patients were initially selected based on clinical signs of left-sided sclerosis, but (as described below) some also showed various degrees of right sclerosis, which allowed us to test the effect of damage to either side of the amygdala. Matched healthy controls also participated. During event-related fMRI, patients and controls were presented with faces with fearful or neutral expressions at task-relevant or task-irrelevant positions. Our findings provide the first direct demonstration that structural damage to the amygdala (but not to the hippocampus) eliminates the increased activation that normally occurs in the visual cortex in response to emotional facial expressions, but does not affect the enhanced fusiform-cortex activity linked to voluntary attention to faces based on task relevance. We also show by voxel-based volumetry that amygdala damage has a functional impact on the distant visual cortex despite the latter remaining structurally intact.

\section{RESULTS}

We studied 13 patients who had sclerotic damage to both the amygdala and the hippocampus (the AH group); 13 patients who had sclerotic damage limited to the hippocampus, sparing the amygdala ( $\mathrm{H}$ group);

${ }^{1}$ Laboratory for Neurology and Imaging of Cognition, Departments of Neurology and Neurosciences, Centre Médical Universitaire, University of Geneva, 1211 Geneva 4 , Switzerland. ${ }^{2}$ Institute of Cognitive Neuroscience \& Department of Psychology, University College London, Alexandra House, 17 Queen Square, London WC1N 3AR, UK. ${ }^{3}$ Epilepsy Imaging Group, Department of Clinical and Experimental Epilepsy, Institute of Neurology, 33 Queen Square, London WC1N 3BG, UK. ${ }^{4}$ Douglas Hospital Research Centre, McGill University, 6875 LaSalle Blvd., Montreal, Quebec H4H 1R3, Canada. ${ }^{5}$ Wellcome Department of Imaging Neuroscience, University College London, 12 Queen Square, London WC1N 3BG, UK. Correspondence should be addressed to P.V. (patrik.vuilleumier@medecine.unige.ch).

Published online 24 October 2004; doi:10.1038/nn1341 


\section{ARTICLES}

Table 1 Demographic and behavioral data for each group of participants (mean \pm s.d.)

\begin{tabular}{|c|c|c|c|c|c|c|c|}
\hline \multirow[b]{2}{*}{ Age } & \multicolumn{2}{|c|}{$\mathrm{A}+\mathrm{H}$} & \multicolumn{2}{|c|}{$\mathrm{H}$ only } & \multicolumn{2}{|c|}{$\mathrm{N}$ controls } & \multirow[b]{2}{*}{ n.s. } \\
\hline & 34.8 & $( \pm 9.4)$ & 36.7 & $( \pm 8.4)$ & 35.9 & $( \pm 8.6)$ & \\
\hline Male/female & $7: 6$ & & $5: 8$ & & $7: 6$ & & \\
\hline $\mathrm{R} / \mathrm{L}$ handedness & $12: 1$ & & $12: 1$ & & $12: 1$ & & \\
\hline L hippocampus sclerosis T2 intensity (ms) & 95.5 & $( \pm 1.2)$ & 93.6 & $( \pm 3.6)$ & 86.6 & $( \pm 2.5)$ & $\mathrm{AH}=\mathrm{H}>\mathrm{N}\left({ }^{*}\right)$ \\
\hline $\mathrm{R}$ hippocampus sclerosis $\mathrm{T} 2$ intensity (ms) & 87.7 & $( \pm 1.5)$ & 86.7 & $( \pm 1)$ & 86.6 & $( \pm 2.5)$ & $\mathrm{AH}=\mathrm{H}=\mathrm{N}$ \\
\hline $\mathrm{L}$ amygdala sclerosis $\mathrm{T} 2$ intensity (ms) & 94.5 & $( \pm 1.6)$ & 88.1 & $( \pm 1.8)$ & 88 & $( \pm 2)$ & $A H>H(*) ; H=N$ \\
\hline $\mathrm{R}$ amygdala sclerosis $\mathrm{T} 2$ intensity (ms) & 89.2 & $( \pm 2.9)$ & 85.9 & $( \pm 2.3)$ & 88 & $( \pm 2)$ & $\mathrm{AH}>\mathrm{H}(* *) ; \mathrm{H}=\mathrm{N}$ \\
\hline L hippocampus volume $\left(\mathrm{mm}^{3}\right)$ & 1932 & $( \pm 384)$ & 1807 & $( \pm 414)$ & - & - & n.s. \\
\hline R hippocampus volume $\left(\mathrm{mm}^{3}\right)$ & 2964 & $( \pm 383)$ & 2647 & $( \pm 423)$ & - & - & n.s. \\
\hline Cranial volume $\left(\mathrm{cm}^{3}\right)$ & 155 & $( \pm 22)$ & 147 & $( \pm 15)$ & - & - & n.s. \\
\hline Age at onset (years) & 6.6 & $( \pm 4.6)$ & 7.2 & $( \pm 7.8)$ & - & - & n.s. \\
\hline No. of seizures/month & 6.2 & $( \pm 5.9)$ & 7.6 & $( \pm 9.9)$ & - & - & n.s. \\
\hline No of drugs/day & 2.23 & $( \pm 0.9)$ & 2.15 & $( \pm 0.8)$ & - & - & n.s. \\
\hline Total drug dose (mg) & 826 & $( \pm 898)$ & 898 & $( \pm 906)$ & - & - & n.s. \\
\hline WAIS-R IQ-P & 102.7 & $( \pm 16.7)$ & 98.6 & $( \pm 15.7)$ & - & - & n.s. \\
\hline WAIS-R IQ-V & 95.7 & $( \pm 12.9)$ & 90.3 & $( \pm 9)$ & - & - & n.s. \\
\hline \multicolumn{8}{|l|}{ Behavior during fMRI: } \\
\hline Accuracy (\% correct) & 73.6 & $( \pm 18.1)$ & 69.0 & $( \pm 20.7)$ & 72.3 & $( \pm 19.3)$ & n.s. \\
\hline Reaction time (ms) & 975 & $( \pm 186)$ & 985 & $( \pm 250)$ & 874 & $( \pm 224)$ & n.s. \\
\hline
\end{tabular}

${ }^{*} P \leq 0.001,{ }^{* *} P \leq 0.02$.

and 13 normal volunteers ( $\mathrm{N}$ group) who were matched to the demographic characteristics of the affected patients (Table 1 and Methods). Amygdala and/or hippocampal sclerosis was assessed by tissue intensity on structural MRI, using conventional spin-echo dual echo images known to provide reliable quantitative assessment of sclerotic lesions in medial temporal lobe structures ${ }^{24,25}$. The amygdala and/or hippocampus were classified as abnormal when average tissue intensity, measured using a standard procedure ${ }^{24,28}$ by T2 MRI values throughout the volume of either structure, was at least 2 s.d. above the mean of normal controls (see Methods). ANOVA on these T2 values showed a significant triple interaction between following factors: group $(\mathrm{AH}$ versus $\mathrm{H}$ ), region (amygdala versus hippocampus) and hemisphere (right versus left) $\left(F_{1,24}=8.45, P=0.008\right)$. As expected, both the $\mathrm{AH}$ and $\mathrm{H}$ groups showed higher $\mathrm{T} 2$ intensity, indicative of sclerosis, in the left hippocampus compared to their own right hippocampus or to the hippocampi of the healthy population $\left(t_{12}>9.21, P<0.001\right.$; see Table 1). Hippocampal sclerosis did not differ between $\mathrm{AH}$ and $\mathrm{H}$ patients $\left(t_{24}=1.15\right.$, nonsignificant $)$. However, $\mathrm{AH}$ patients showed higher T2 intensity in the left amygdala $\left(t_{12}=5.61, P<0.001\right)$. The $\mathrm{H}$ group did not $\left(t_{12}=1.09\right.$, nonsignificant), as damage to the subjects in this group was restricted to the hippocampus. The severities of hippocampal and amygdala sclerosis were not correlated $(r=0.275$, $P=0.18$; ref. 26). The average T2 signal in the right amygdala was also higher in AH patients than in $\mathrm{H}$ patients $\left(t_{24}=2.41, P=0.024\right)$, despite initial clinical selection on the basis of left-sided sclerosis only (see Methods), indicating some degree of bilateral structural losses. This allowed us to examine the role of structural lesions parametrically on either the left or right side (see below). Note that factors other than amygdala damage (temporal sclerosis and medical history) were controlled for by comparing the $\mathrm{AH}$ and $\mathrm{H}$ groups (Table 1), thus minimizing any general effects due to temporal lobe disease and epilepsy $y^{29,30}$. All groups had structurally intact cortex outside the medial temporal lobe. Indeed, in a direct voxel-by-voxel morphometric analysis ${ }^{31,32}$, no other brain region showed significant differences in gray-matter volume between patient groups (all $P>0.05$ corrected), as confirmed in more detail below for visual areas.
To determine the functional impact of amygdala damage on distant, structurally intact areas, we exploited an event-related fMRI paradigm previously used in healthy volunteers ${ }^{11}$. We manipulated emotional expression (fearful or neutral) on the faces displayed to the subjects and manipulated the subjects' focus of attention (faces being either attended or ignored), and used these conditions as orthogonal factors in a $2 \times 2$ design. On each trial, pictures of two faces and two houses were briefly shown together $(250 \mathrm{~ms})$. On any given trial, both faces had the same expression: either neutral or fearful, in a randomized sequence (Fig. 1). Participants were told before each of the two blocks of trials to focus on just one pair of stimuli at a time (either the vertical or the horizontal pair) and to judge whether they were the same or different. Because the target of focus and the facial expressions were randomized across trials, participants could not anticipate a particular type of stimulus or emotion. Behavioral performance during fMRI did not differ between groups (Fig. 1 and Table 1).

\section{Modulation of visual cortex by selective attention}

We first examined the effect of attention on fMRI, comparing, in each patient group separately, the trials in which participants judged faces only versus the trials in which they judged houses only. As expected $^{11,33}$, bilateral fusiform regions were activated in all groups when the focus of attention was on the faces (Fig. 2a-c and Table 2). These data confirm that participants attended selectively to the stimuli as instructed, reliably activating the fusiform cortex for task-relevant faces. These fusiform activations did not differ between groups, as confirmed by a voxel-by-voxel comparison across the whole brain for all 39 participants: no area showed significant group $\times$ attention interaction, except for greater activation of the left entorhinal and parahippocampal cortices in $\mathrm{N}$ compared to $\mathrm{AH}$ and $\mathrm{H}$, consistent with medial temporal sclerosis ${ }^{32}$ ( $x$ y $z$ coordinates $=-21-21-21$ and -27 $-39-21$, respectively, $F>11.2, P<0.001)$.

\section{Modulation of visual cortex by selective attention}

We next examined the main effect of emotion in each group separately, comparing the effect of fearful faces in all trials to that of neu- 
Figure 1 Sample visual stimuli. Two faces and two houses appeared on each trial, with each pair showing either the same items (half of all trials) or different items from the same category (other half of trials). Faces were either vertically or horizontally aligned (half of trials each), and both bore the same expression in each trial (fearful or neutral). In one block of trials, participants judged only the vertical pair (same/different) while ignoring horizontal stimuli, whereas in another block of trials, they judged only the horizontal pair while ignoring vertical stimuli (64 trials each). Block order was counterbalanced across participants and groups. An ANOVA on accuracy and reaction times showed similar performance across $\mathrm{AH}, \mathrm{H}$ and $\mathrm{N}$ (see Table 1; mean $71.6 \%$ correct, mean reaction time 945 ms; no group effects, $F_{2,36}=0.34$ and 0.86 , respectively). There was an effect of attended stimulus category (more errors and slower responses for faces versus houses: $67 \%$ versus $77 \%$ correct, 1,004 ms versus $886 \mathrm{~ms}, F_{1,36}=16.6$ and 24.1 , respectively; $P<0.001$ ), but none related to emotion (for fearful versus neutral faces in the display, $71 \%$ versus $73 \%$ scored correctly, reaction times were 958 ms versus $930 \mathrm{~ms}, F_{1,36}=0.61$ and 2.57, respectively, $P>0.12$ ). There was no interaction between attention and emotion or between either of these and the group factor in behavior. Eye position was recorded throughout scanning (Applied Science Laboratory, Bedford, Massachusetts) and showed no systematic differences between conditions or groups (data available for offline analysis in seven participants in each group; ANOVA on mean displacement during a 250-ms period after stimulus onset, with factors of group, attended location, attended category and emotion: all $F$ values $<2.4, P>0.10$ ).

tral faces, irrespective of the spatial attention of the subjects. This gave rise to activation of the right amygdala in all three groups, regardless of whether faces were task relevant or not (confirming previous findings related to this paradigm ${ }^{11}$; see Table 2 and Supplementary Fig. 1 online). Emotional expressions had a similar effect on the left amygdala but in $\mathrm{N}$ and $\mathrm{H}$ patients only, consistent with the major left amygdala damage in group $\mathrm{AH}$.

Our main question concerned how brain areas distant from but connected to the amygdala, such as the visual cortex, would respond to fearful faces. As expected ${ }^{8,9,11}$, the healthy $\mathrm{N}$ group showed significantly higher bilateral activity in the fusiform and extrastriate cortices in response to fearful versus neutral faces (Fig. 2d and Table 2), consistent with the enhanced visual processing of emotional stimuli ${ }^{13}$. A similar pattern of increased activation in response to fearful faces versus neutral faces was found in $\mathrm{H}$ patients in the (predominantly left) fusiform cortex, as well as in other areas (Fig. 2e and Table 2). AH patients, however, showed no effect in the fusiform regions, not even at a low statistical threshold $(P<0.05$ uncorrected). They showed only a weak increase in response to fearful faces in the left posterior inferior temporal gyrus (Fig. $2 \mathrm{f}$ and Table 2). Thus, amygdala sclerosis in $\mathrm{AH}$ patients abolished the enhanced neural responses to fearful faces normally seen in distant fusiform areas, and also found here for both healthy controls and for patients in the H group.

This difference between groups in visual activation in response to fearful faces was confirmed by three further analyses. First, we analyzed in each group the main effect of emotional versus neutral faces within those fusiform voxels responding to task-relevant faces (as determined by the orthogonal main effect of attention). Fusiform activity was significantly enhanced by fearful expressions for the $\mathrm{N}$ group in both hemispheres and across both attentional conditions (that is, with faces in a task-relevant or task-irrelevant position; Fig. $2 \mathrm{~g}, \mathrm{~h}$ ). The $\mathrm{H}$ group showed similar fusiform enhancement, greater in the left hemisphere (Fig. 2i) than in the right (see Table 2). By contrast, AH patients showed only a weak response to emotion, and in just a single voxel of face-responsive regions in the posterior left temporal cortex (Fig. 2j), with no significant effect in the fusiform cortex on either side (not even at threshold of $P<0.05$ uncorrected).

Second, we performed a voxel-wise analysis across the whole brain for all 39 participants to identify any areas in which activation by fearful versus neutral faces differed as a function of group (that is, for which there was an interaction between group and the main effect of emotion). This revealed significant differences in bilateral temporal regions (Table 2, and Fig. 3), including in the bilateral fusiform and left occipital cortices, as well as in the right superior temporal sulcus and in the right anterior cingulate cortex. In all these regions, the interaction was a result of greater activation by fearful faces for the $\mathrm{N}$ and $\mathrm{H}$ groups than for the $\mathrm{AH}$ group (Table 2, and Fig. 3e,f). Note that this bilateral loss in visual enhancement of cortex activation for $\mathrm{AH}$ patients is consistent with the fact that, as a group, they showed higher T2 sclerosis values in the amygdala on both sides (see above), even though they were initially selected based on left-side sclerosis only.

\section{Ipsilateral amygdala-cortical modulations}

Finally, and notably, we performed a parametric analysis to identify brain regions, in individual subjects, where activation by fearful versus neutral faces differed in direct proportion to the severity of structural amygdala sclerosis on either side. Instead of splitting affected patients into two different groups as before $(\mathrm{H}$ versus $\mathrm{AH})$, we now pooled them all together in a multiple regression analysis. Statistical parametric mapping (SPM) contrasts from the main effect of fearful minus neutral faces were computed for each participant as a dependent variable, while structural T2-intensity values in the amygdala or hippocampus (left or right side separately) for each individual served as four independent predictors within a single regression model (see Methods). This allowed us to test for regions across the whole brain where differential responses to fearful minus neutral faces were attenuated as a linear function of increased structural sclerosis (as indexed by $\mathrm{T} 2$ intensity ${ }^{24,28}$ ) in either the amygdala or hippocampus. When examining the effect of the left amygdala $\mathrm{T} 2$ values, we found a significant relationship between the amount of sclerosis and reduced emotional activation in the visual cortex, particularly in the left posterior fusiform (Fig. 4a) and left occipital cortices $(-3-993, T=3.38$, $P<0.001)$. A similar relationship was found in the left medial hypothalamus (Fig. 4c), left retrosplenial cortex $(-12-4821, T=3.91$, $P<0.001)$, left hippocampus $(-39-24-12, T=4.02 P<0.001)$, bilateral anterior cingulate cortex (15 57 0, $-957-9, T>3.58, P<0.001)$, right parietal operculum $(48-624, \mathrm{~T}=3.67, P<0.001)$ and right superior temporal sulcus $(42-4818, T=2.86, P<0.005)$. Likewise, when we examined the effect of right amygdala $\mathrm{T} 2$ values in the same parametric analysis, we also found increased sclerosis to correlate 


\section{ARTICLES}

Figure 2 Activation by attention and emotion. (a-c) SPMs showing main effects of attention (faces $>$ houses at task-relevant locations, irrespective of emotion), overlaid on the mean anatomical scan for each group (threshold $P<0.01$ for illustration). Activation of fusiform cortex by attention to faces was found similarly in all three groups. (d-f) SPMs showing main effects of emotion (fearful > neutral faces, irrespective of attention). Activation of fusiform and posterior temporal cortex by fear was found in normal controls (d) and in patients with hippocampal damage only (e), but not in patients with amygdala damage (f; see dotted ellipses). (g-j) Average parameter estimates of activity $( \pm$ s.e.m.) showing effects of emotion in faceresponsive regions of fusiform cortex, plotted for all conditions in each group. Volumes of interest in fusiform were defined by the main effect of attention (faces $>$ houses, threshold at $P<0.05$ ) and then inspected for activation by emotional expression. In healthy group $\mathrm{N}$, fearful expressions produced significant increases $(\mathbf{g})$ in right anterior fusiform (96 voxels showing joint effects of attention and emotion; peak coordinates $39-45-24, T=4.33, P<0.05$ corrected for small volume of interest) and (h) in two left fusiform regions (total 13 voxels activated; anterior peak coordinate $-42-78-9$, $\mathrm{T}=2.88$; anterior peak coordinates $-45-45-21$, $\mathrm{T}=2.74 ; P<0.007$ uncorrected). (i) In patients with left hippocampal damage only (H group), fearful expressions also increased activity in two regions of left fusiform (total 39 voxels; anterior peak coordinates $-36-60-21, T=5.41$; posterior peak coordinates $-27-75-12, T=3.44 ; P<0.05$ corrected for small volume). (j) In patients with additional damage to left amygdala ( $\mathrm{AH}$ group), fearful expressions produced significant increases in just one single face-selective voxel of posterior inferior temporal gyrus $(-42-72-21, \mathrm{~T}=3.53, P=0.002$ uncorrected). There was no significant fear-related increases in fusiform in this group, including left anterior fusiform activated by attention to faces (maximum fear trend found at coordinates $-45-60-24, T=1.66, P=0.06$ uncorrected). Fear-related effect were similarly found in posterior right fusiform for $\mathrm{H}$ but not $\mathrm{AH}$ patients (see Table 2). a Healthy controls
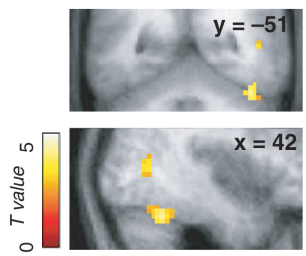

d
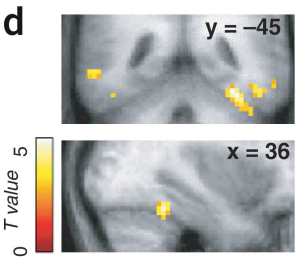

e

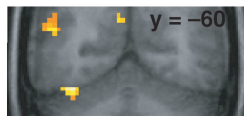

g

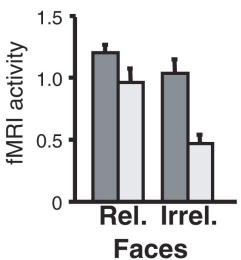

h

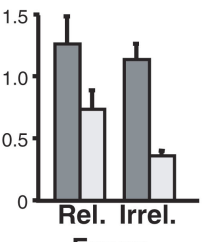

i

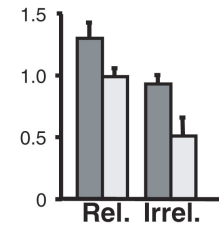

Faces

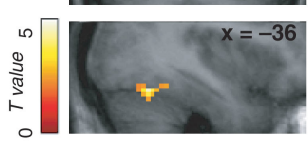

C $\mathrm{A}+\mathrm{H}$ damage
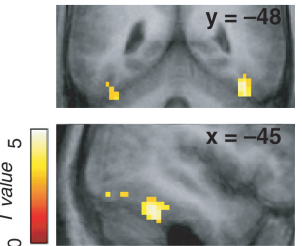

f
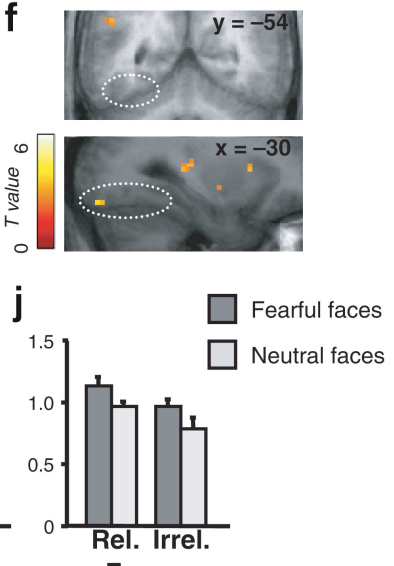

Faces with reduced emotional responses in visual cortex, but this time in the other (but once again ipsilateral) hemisphere: in the right posterior fusiform cortex (Fig. 4b) and the right peristriate cortex (12 -84 $24, T=3.18, P=0.002)$. These were almost mirror images of the lefthemispheric effects that correlated with left amygdala T2 sclerosis (Fig. 4a). Thus, although the subjects were initially clinically selected based on left sclerosis (see Methods), our results show that the varying degrees of sclerosis of the right amygdala had a significant impact, exerting a parametric effect on visual activation by emotional faces within the same ipsilateral hemisphere.

These data indicate that, across all subjects, the degree of differential activation in the visual cortex by faces with fearful expressions was reduced by the amount of sclerotic amygdala tissue within the ipsilateral hemisphere. We found no such parametric correlation of emotional activation in distant brain areas to structural $\mathrm{T} 2$ values in the left or right hippocampus, confirming that our results are specific to amygdala sclerosis. We also performed further regression analyses including independent covariates for amygdala and hippocampal T2 sclerosis, plus two other covariates to model any interaction due to coexisting lesions in both structures ${ }^{34}$, by combining together the T2 values from both regions on each side (see Methods). This again confirmed the selective impact of amygdala sclerosis on visual areas, without any differential effect owing to sclerosis in both the amygdala and the hippocampus (which showed a correlation only with entorhinal-cortex activity on both sides, and with activity in right retrosplenial regions). Figure $4 \mathrm{~d}$ illustrates the inverse relationship between the effect of emotion on the left fusiform cortex and left amygdala (but not hippocampal) T2 sclerosis intensity for trials with task-relevant as well as task-irrelevant faces (see also Supplementary Fig. 2 online).

Our parametric analyses thus identify a network of brain regions (including the fusiform cortex) in which an increased response to fearful, as compared to neutral, faces depends on the integrity of the amygdala. The greater the degree of amygdala sclerosis, the smaller the differential response to fearful faces in all these distant areas. We do not believe that medial temporal lobe disease was responsible for this influence, as neither hippocampal sclerosis alone, nor an interactive combination of amygdala-and-hippocampal sclerosis, produced any parametric effect on cortex responses to emotional visual stimuli.

Although the effect of fearful faces on the bilateral fusiform and temporo-occipital areas was eliminated in the $\mathrm{AH}$ group, these visual areas were nevertheless structurally intact, as shown by normal modulation during attention to faces and by structural analysis. Indeed, voxel-by-voxel morphometry ${ }^{31,32}$ applied to the visual regions showing a distant functional response to amygdala damage (see above and Methods) revealed no differences in gray-matter density between patient groups (even at threshold of $P<0.05$, uncorrected). Moreover, no correlation was found between left- or right-amygdala T2 and gray-matter density in these visual regions.

\section{DISCUSSION}

Our study demonstrates that an intact amygdala is necessary for the enhanced activation of distant areas-including face-responsive regions in the extrastriate cortex and early occipital areas-in 
ARTICLES

Table 2 SPM peaks of activation for each group

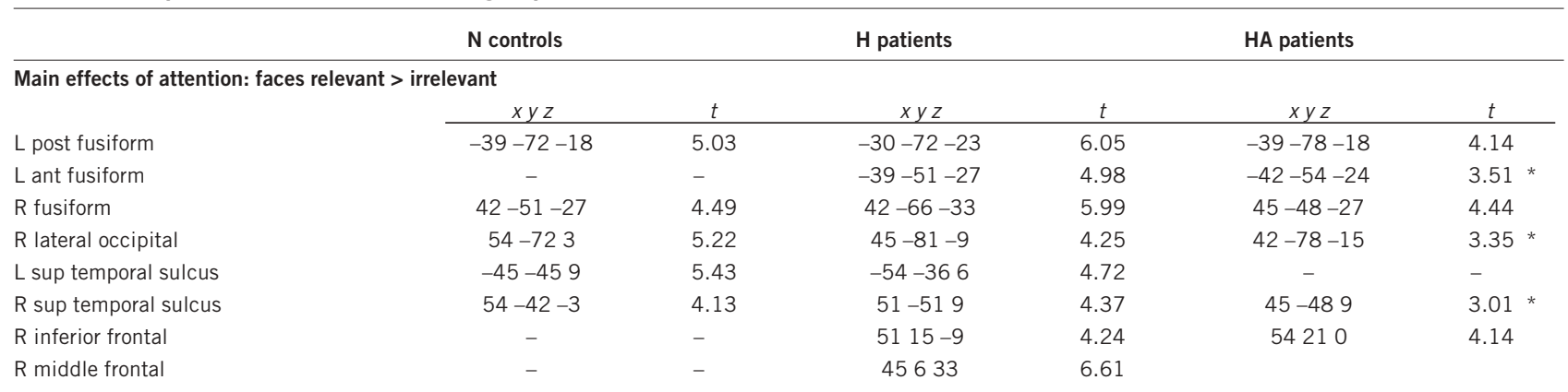

Main effect of emotion: fearful > neutral faces

$L$ fusiform

$L$ inferior temporal

$\mathrm{L}$ middle temporal

$\mathrm{L}$ peristriate

R fusiform

$\mathrm{R}$ sup temporal sulcus

$\mathrm{R}$ amygdala

$\mathrm{L}$ amygdala

$\mathrm{R}$ insula

$L$ insula

$L$ inferior frontal

Group differences for emotion

L striate

$L$ post fusiform

$L$ medial fusiform

$L$ ant fusiform

L sup occipital

L lateral occipital

$L$ lingual

$R$ medial fusiform

$\mathrm{R}$ ant fusiform

$R$ post fusiform

$\mathrm{R}$ lat occipital

$R$ lingual

$\mathrm{R}$ sup temporal sulcus

$\mathrm{R}$ rostral cingulate

\begin{tabular}{cc}
$x y z$ & $t$ \\
\hline$-54-63-18$ & 3.69 \\
$-39-66-3$ & 5.26 \\
$-60-51-6$ & 4.32 \\
$-15-7227$ & 3.78 \\
$33-45-18$ & 5.15 \\
$54-42-18$ & 4.45 \\
$24-3-21$ & 4.29 \\
$-15-3-21$ & 3.62 \\
$3312-15$ & 5.63 \\
$-3912-15$ & 3.99 \\
- & -
\end{tabular}

$-$

$x y z$
$-36-60-21$
$-27-75-12$
$-45-6015$
$-21-9015$
$39-78-18$
$48-42-9$
$33-9-27$
$-18-9-18$
$480-6$
2499
-

ANOVA: interaction fear effect $\times$ group

\begin{tabular}{ccl}
$t$ & $x y z$ & \multicolumn{1}{c}{$t$} \\
5.41 & - & - \\
$3.44 *$ & $-30-90-18$ & 3.88 \\
4.20 & - & - \\
5.44 & - & - \\
$3.26 *$ & - & - \\
$3.37 *$ & - & - \\
5.06 & $16-12-24$ & 3.79 \\
$2.37 *$ & - & - \\
$3.06 *$ & $513-6$ & 4.35 \\
3.87 & - & - \\
- & -48246 & 6.17
\end{tabular}

Coordinates $(x y z)$ are given in MNI space. For $t$-test contrasts, all $P \leq 0.001$ uncorrected except $* P \leq 0.005, \# P \leq 0.01$ (random effects). For ANOVA $F$-tests, all $P \leq 0.01$, except ${ }^{*} P \leq 0.05$.

response to faces with fearful expressions. Amygdala lesions can thus change the functional pattern of activation to emotional stimuli in regions that are distant from the amygdala itself. This provides the first direct evidence supporting previous speculation that enhanced activation to fearful stimuli in these distant sensory regions depends on amygdala function ${ }^{9,13,14,20-22}$. Although temporal lobe disease is a complex and variable medical condition ${ }^{30}$, our comparison of patients with $\mathrm{AH}$ and $\mathrm{H}$ damage (in addition to our analyses of parametric structural loss) helped us control for potential confounding factors stemming from the presence of temporal-lobe disease or epilepsy and from treatment.

We were further able to show that the influence of the structural integrity of the amygdala on functional activations in the visual cortex operates predominantly via ipsilateral connections. This was clearly indicated by the parametric correlation between emotional enhancement in the left or right extrastriate areas, with T2 sclerosis intensity in left or right amygdala, respectively. These data suggest that the emotional modulation of visual areas by the amygdala is organized in a largely symmetrical manner in both hemispheres. These results are consistent with anatomical studies of connectivity in monkeys ${ }^{20,21}$ and humans ${ }^{35}$ showing direct projections from the amygdala to many ipsilateral areas along the cortical visual pathways, including to the striate and temporal cortex. Our work expanded upon these studies, directly demonstrating that these projections had a functional significance. Previous animal studies provided only indirect evidence, including enhanced activity of face-selective neurons in the temporal cortex in response to emotional facial expressions $^{13}$, or deficits in visual behavior and learning after lesions were 


\section{ARTICLES}
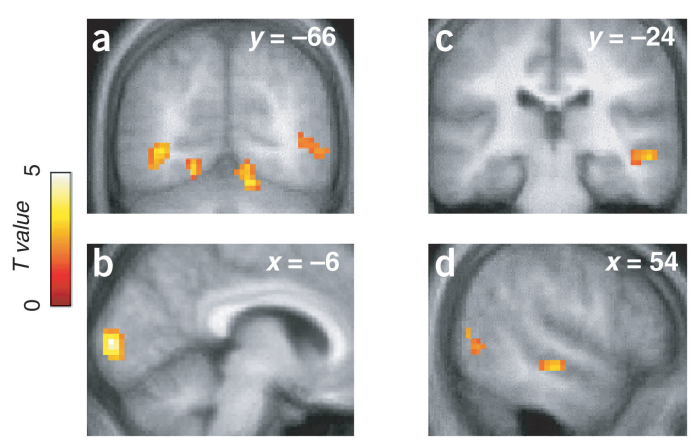

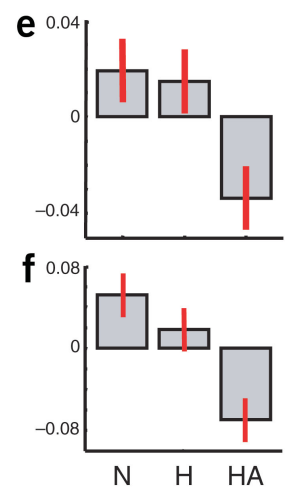

Figure 3 SPMs of emotion $\times$ group interaction (threshold $P<0.01$ for illustration). (a-d) ANOVA across the whole brain showed that the main effect of fearful versus neutral faces differed between groups in (a) bilateral fusiform and lateral occipitotemporal areas (b) left striate occipital cortex, and (c,d) right superior temporal sulcus (STS). (e,f) Parameter estimates of the relative size of effect in this ANOVA (arbitrary units, meancentered) for peaks in (e) left fusiform $(-36-69$ -9) and (f) left striate cortex (-6 -99 3), showing increased activation to fearful expressions in both $\mathrm{N}$ controls and $\mathrm{H}$ patients, but not $\mathrm{AH}$ patients. Right STS showed the same pattern. made in the amygdala ${ }^{18,23,36}$. Our new results support proposals ${ }^{21}$ that one function of amygdala projections is to enhance sensory processing of emotional stimuli. Further, although previous neuroimaging studies of healthy subjects have reported increased activation of the visual cortex in response to fear-related stimuli ${ }^{8,11}$, here we show for the first time that this activation depends on the integrity of the amygdala, and that it therefore truly results from emotional evaluation of the stimuli, rather than simply relating to particular visual features that might be extracted in a feedforward manner within visual cortex itself. The sites of emotional modulatory effects within the striate and fusiform cortices were very similar in our study to those found in previous reports $9,11,37$.

Our orthogonal manipulation of attention confirmed that the influence of the amygdala on the fusiform cortex can be dissociated from modulation by attention in our paradigm ${ }^{11}$ (but see ref. 37 for different task conditions). The effects of expression and attention were additive in controls and $\mathrm{H}$ patients, and amygdala damage in $\mathrm{AH}$ patients affected only the fear-related activations, not the attention effect. These amygdala influences, which can boost visual processing of fearful stimuli, provide a plausible neural substrate for the prioritization of emotional stimuli over neutral stimuli in perception ${ }^{3-5}$ and for the formation of subsequent memories ${ }^{1,2,17,22,38}$.

However, this amygdala modulation of visual processing is presumably not necessary for explicit recognition of fearful expressions ${ }^{12}$, because such judgments are not consistently impaired in humans with unilateral amygdala lesions, even when these lesions are more extensive than the focal sclerosis in our subjects ${ }^{39-42}$. Owing to clinical constraints, it was not possible to test all of the present patients on explicit behavioral judgments of emotion. However, several of our patients underwent more precise tests (P. Rotshtein and P. Winston, personal communication) using a facial-expression classification task $^{40}$ (the

Figure 4 SPM parametric analysis. (a) Higher T2 intensity of sclerosis in left amygdala was inversely related to the main effect of fearful minus neutral faces in distant left posterior fusiform cortex $(-24-78-15$, $\mathrm{T}=3.66, P=0.001$ ). (b) Symmetrically, higher T2 intensity in right amygdala was inversely related to the effect of fearful expressions in distant right posterior fusiform cortex (36 -71 $-15, T=3.47, P=0.001$ ). (c) A similar correlation between higher left amygdala $\mathrm{T} 2$ intensity and reduced activation to fearful faces was found in left medial hypothalamus $(-6-12$ $-15, T=3.51, P=0.001$ ) and other regions connected to the amygdala (see text). (d) Difference in activation to fearful minus neutral faces in left fusiform plotted against T2 sclerosis intensity in left amygdala across the 26 patients ( $\mathrm{AH}$ and $\mathrm{H}$ ), showing a reliable correlation both when faces were task-relevant $(R=-0.29)$ and when task-irrelevant $(R=-0.38)$. There was no such correlation with T2 sclerosis intensity in left hippocampus (0.09 and -0.06 , respectively). See also Supplementary Figure 2. remaining patients had already proceeded to surgery). In line with previous studies showing no effect of unilateral focal amygdala lesions on explicit judgments of expression $39,40,42$, none of these AH patients exhibited any significant impairment with respect to explicit recognition of facial expression (correct choice for fearful $=$ mean $70 \% \pm 10 \%$ in $4 \mathrm{AH}$ patients, versus $64 \% \pm 6 \%$ in 8 controls; total correct for all 6 basic expressions $=77 \% \pm 5 \%$ in $\mathrm{AH}, 75 \% \pm 2 \%$ in controls; all $t<$ $1.22, P>0.25$ (P. Rotshstein and P. Winston, personal communication)). Moreover, unilateral amygdala lesions may not impair conscious subjective emotional experiences either ${ }^{43}$. We therefore suggest that the enhancement of sensory responses by the amygdala (as shown here for the visual cortex) might be more critical for eliciting adaptive allocation of processing resources based on affective values ${ }^{4,14}$, and for boosting perceptual analysis and memory of threat stimuli ${ }^{15,21,38}$, rather than for explicit appraisal of facial emotion. Future studies could adapt our approach to examine how modulation of distant visual areas by the amygdala might contribute to subsequent enhanced memory for faces with affectively salient expressions ${ }^{38,44,45}$.

In our study, amygdala damage also reduced differential responses to fearful faces in other brain regions beyond the visual cortices, including in the left medial hypothalamus, the retrosplenial cortex, and the hippocampal region. All are directly connected with the amygdala, forming a coordinated network regulating responses to emotional events ${ }^{17,46}$. Amygdala sclerosis also reduced emotional
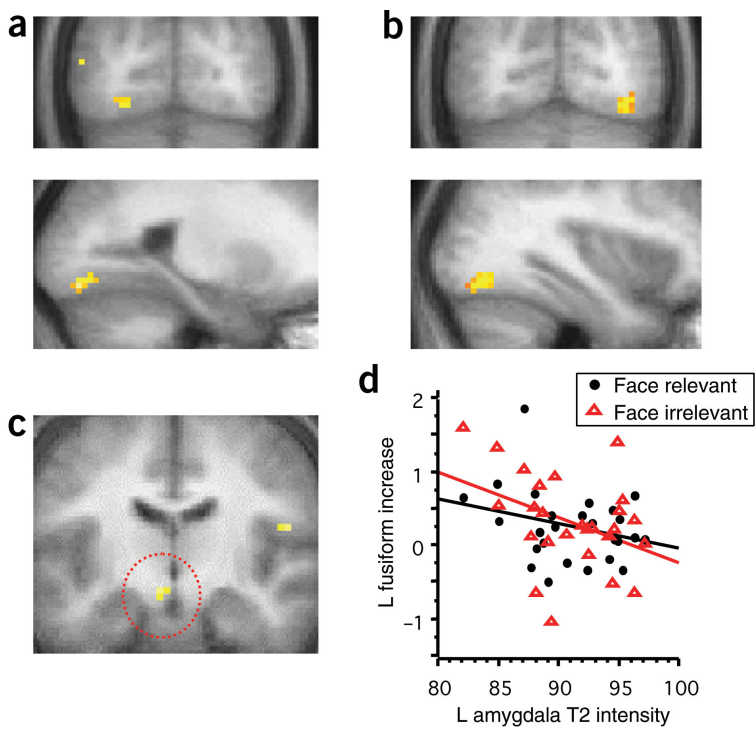
responses in the right superior temporal sulcus (STS) and rostral cingulate cortex, which have previously been implicated in emotional and social processing ${ }^{47}$. Our new findings indicate that activation in response to emotional faces in these regions also depends on amygdala integrity, rather than just on local processing alone.

In conclusion, through fMRI of patients with focal temporal lesions and appropriate controls, we showed that amygdala damage disrupts functional activation by emotional stimuli of distant, structurally intact but connected areas of the brain, notably the visual cortex. This reveals a distant functional influence of the amygdala on posterior sensory cortices, and provides a mechanism for prioritized processing of fear-related stimuli. Such influences were first hypothesized on purely anatomical $^{20,21}$ or behavioral ${ }^{4}$ grounds, but were directly shown here for the first time through the functional effects of structural amygdala damage on neural activity in distant areas. More generally, we show that fMRI can be exploited to assess interactions between interconnected brain systems, revealing how a focal lesion to a 'higher-level' structure such as the amygdala can affect the function of other areas, including the 'lower-level' visual cortex. The lesion approach can therefore be usefully combined with functional imaging techniques to examine the functional influences of distant brain regions.

\section{METHODS}

Patients. All patients $(n=26)$ were clinically tested, before surgical treatment, for chronic epilepsy due to medial temporal lobe sclerosis. Healthy controls were chosen from among groups that included spouses, partners and friends, to match each patient's age and socioeconomic status. Controls had no history of neurological or psychiatric illness, and none was included in our previous work ${ }^{11}$. Patients were initially selected for unilateral left temporal sclerosis in order to generate a sufficiently large sample of subjects with well-defined lesions; because other ongoing projects were examining left-hemisphere verbal functions (results not reported here); and because predominant activation of the left amygdala had been observed in normal subjects in a previous study using the same paradigm ${ }^{11}$.

Some patients also showed varying degrees of structural loss in the right amygdala (leading to right-amygdala T2 values that were higher, on average, for the AH group than for the $\mathrm{H}$ group). This allowed us to test for distinct effects of left- or right-amygdala damage in this patient sample (see parametric analyses).

The participants in the $\mathrm{AH}, \mathrm{H}$, and $\mathrm{N}$ groups $(n=13$ each) included 6,8 , and 6 females, respectively, and had a mean age 35.8; all but one subject in each group were right-handed. The $\mathrm{AH}$ and $\mathrm{H}$ patient groups did not differ statistically in age, duration of epilepsy, type and frequency of seizures, drug regimen, neurological status, and general cognitive functions (see Table 1). None of the patients had a seizure around the time of our investigations. Clinical and neuroradiological diagnosis of left medial temporal sclerosis was made prior to our study by independent physicians. Several patients participated in a behavioral study (P. Rotshstein \& P. Winston, personal communication) following our investigation where they showed no significant recognition deficit (see main text) for basic facial expressions (fear, surprise, anger, sadness, disgust, happiness; from ref. 48), by pointing to the appropriate label out of six choices ${ }^{40}$. All participants had normal or corrected vision, and gave informed consent according to procedures approved by the Ethical Committee of the National Hospital for Neurology and Neurosurgery, London.

Structural MRI T2 measures. To identify amygdala and hippocampus sclerosis, T2 relaxation times were measured using spin-echo dual-echo sequences on a 1.5-T MR scanner (GE), in both patients and healthy controls, as part of a standard clinical protocol used to assess temporal lobe epilepsy ${ }^{24,28}$. Hippocampal T2 values were measured from regions of interest drawn on contiguous coronal 5-mm slices throughout the extent of each hippocampus. Amygdala T2 values were measured on the more anterior slices rostral to the hippocampus, according to previously described procedures ${ }^{24,25}$. T2 values provide refined measures of tissue damage and glial sclerosis in medial temporal structures, in addition to the cell loss indexed by volumetry measurements ${ }^{49,50}$. These measurements were made by clinicians independent of our study. We selected patients who had had structural T2 values showing clear- cut abnormalities in the left hippocampus and/or left amygdala ( $>2$ s.d. above the mean for a healthy population). T2 sclerosis intensity in the amygdala and hippocampus were not significantly correlated $(r=0.275, P=0.18)^{26}$. In addition, T2 values from the right temporal lobe allowed a comparison of right and left amygdala sclerosis in the same patients (see text). Detailed clinical and neuroradiological evaluation showed that other brain regions were intact (including formal voxel-based morphometry of visual cortex), and that general cognitive functions were preserved as shown by Wechsler Adult Intelligence Scale (see Table 1).

fMRI scanning and visual stimuli. Stimulus parameters were similar to those in our previous study of healthy subjects ${ }^{11}$. The stimuli included representations of 10 fearful faces ${ }^{47}, 10$ neutral faces from our own database, and 20 houses, with pictures from each category repeated equally across all trials. Average stimulus-onset asynchronies between stimulus events was $4.5 \mathrm{~s}$ (with a range of 2.4-6.8 s), with 32 events for each of the conditions (fearful or neutral faces at relevant or irrelevant locations, irrespective of position).

Data were acquired on a 2T Magnetom VISION system (Siemens, Erlangen, Germany) equipped with a head-volume coil. Functional images were obtained with a gradient echo-planar $\mathrm{T} 2^{*}$ sequence using blood oxygenation level dependency (BOLD) contrast, each comprising 32 contiguous axial slices (2 $\mathrm{mm}$ thick, $3 \times 3 \mathrm{~mm}$ in-plane). Volumes were acquired continuously with a TR of $2.43 \mathrm{~s}$ ( 313 scans total) in a single session ( $13 \mathrm{~min}$ ). Images were processed using SPM99 (www.fil.ion.ucl.ac.uk/spm). Scans were realigned, normalized, slice-time corrected, and spatially smoothed by an 8 -mm full-width half-maximum Gaussian kernel. A high-pass frequency filter (with a cutoff at $120 \mathrm{~s}$ ) and corrections for auto-correlation between scans were applied to the time series. Individual events were modeled by a standard hemodynamic response function. Movement parameters from realignment corrections were entered as covariates of no interest. Parameter estimates of event-related activity were obtained using the general linear model for each voxel in each condition and each subject. A two-stage random-effect analysis was performed using a onesample $t$-test on contrast images obtained for each individual subject. Similarly, individual contrasts from appropriate conditions were entered into a voxel-byvoxel ANOVA with a three-level factor for the groups of subjects $(\mathrm{AH}, \mathrm{H}, \mathrm{N})$ to test for condition $\times$ group interactions. A multiple regression analysis was performed on individual fear-minus-neutral contrast images, using the severity of T2 sclerosis measures in medial temporal lobe (amygdala or hippocampus, right or left) as four independent linear parametric covariates, entered in the same design matrix (further analyses also included two additional covariates modeling any multiplicative interaction of combined lesions in amygdala and hippocampus, considered jointly for each side). For all these second-stage random-effect analyses, resulting SPMs of the $t$-statistic (d.f. $=12$ ) at each voxel were thresholded at $P<0.001$ uncorrected, unless mentioned otherwise. Additional activations at lower thresholds are reported when relevant.

Voxel-based morphometry. After cortical segmentation, gray-matter density in $\mathrm{T} 1$ anatomical scans was compared between patients across the whole brain $^{31}$, and more particularly for all activated visual regions (using a large volume of interest defined by the sum of all brain regions activated by attention or emotion across all groups, at $P<0.05$ uncorrected).

Note: Supplementary information is available on the Nature Neuroscience website.

\section{ACKNOWLEDGMENTS}

Thanks to the patients and their families for participating; to clinicians at the Department of Clinical and Experimental Epilepsy (J. Duncan, L. Sander, M. Walker, H. Cock, S. Sisodiya and M. Koepp) for referring the patients; to P. Bartlett, chief radiographer at the Chalfont Centre for Epilepsy, for providing MRI volume and T2 data; and to P. Rotshtein and J. Winston for providing data from their behavioral testing of the patients. Supported by a Swiss National Science Foundation grant (P.V.), a Fellowship of the Medical Research Council (MPR), a Royal Society-Wolfson Research Merit Award (J.D.) and Wellcome Trust Programme Grants (R.J.D and J.D.).

\section{COMPETING INTERESTS STATEMENT}

The authors declare that they have no competing financial interests.

Received 3 May; accepted 21 September 2004

Published online at http://www.nature.com/natureneuroscience/ 


\section{ARTICLES}

1. Davis, M. \& Whalen, P.J. The amygdala: vigilance and emotion. Mol. Psychiatry 6 , 13-34 (2001).

2. Dolan, R. J. Emotion, cognition and behaviour. Science 298, 1191-1194 (2002)

3. Fox, E. et al. Facial expressions of emotion: are angry faces detected more effi ciently? Cognit. Emotion 14, 61-92 (2000).

4. Anderson, A.K. \& Phelps, E.A. Lesions of the human amygdala impair enhanced perception of emotionally salient events. Nature 411, 305-309 (2001).

5. Vuilleumier, P. \& Schwartz, S. Emotional facial expressions capture attention. Neurology 56, 153-158 (2001).

6. Vuilleumier, P. Facial expression and selective attention. Curr. Op. Psychiatry 15, 291-300 (2002)

7. Morris, J.S. et al. A differential neural response in the human amygdala to fearful and happy facial expressions. Nature 383, 812-815 (1996).

8. Lang, P.J. et al. Emotional arousal and activation of the visual cortex: an fMRI analysis. Psychophysiology 35, 199-210 (1998).

9. Morris, J. et al. A neuromodulatory role for the human amygdala in processing emotional facial expressions. Brain 121, 47-57 (1998).

10. Pizzagalli, D.A. et al. Affective judgments of faces modulate early activity (approximately 160 ms) within the fusiform gyri. Neuroimage 16, 663-77 (2002).

11. Vuilleumier, P., Armony, J.L., Driver, J. \& Dolan, R.J. Effects of attention and emotion on face processing in the human brain: an event-related fMRI study. Neuron 30 829-841 (2001).

12. Vuilleumier, P., Armony, J., Driver, J. \& Dolan, R.J. Distinct spatial frequency sensitivities for processing faces and emotional expressions. Nat. Neurosci. 6, 624-631 (2003)

13. Sugase, Y., Yamane, S., Ueno, S. \& Kawano, K. Global and fine information coded by single neurons in the temporal visual cortex. Nature 400, 869-873 (1999).

14. Armony, J.L., Quirk, G.J. \& LeDoux, J.E. Differential effects of amygdala lesions on early and late plastic components of auditory cortex spike trains during fear conditioning. J. Neurosci. 18, 2646-2652 (1998).

15. Kapp, B.S., Supple, W.F. \& Whalen, P.J. Effects of electrical stimulation of the amygdaloid central nucleus on neocortical arousal in the rabbit. Behav. Neurosci. 108, 81-93 (1994).

16. Dringenberg, H.C., Saber, A.J. \& Cahill, L. Enhanced frontal cortex activation in rats by convergent amygdaloid and noxious sensory signals. Neuroreport 12 , 2395-2398 (2001).

17. LeDoux, J.E. The Emotional Brain (Simon \& Schuster, New York, 1996).

18. Aggleton, J.P. The contribution of the amygdala to normal and abnormal emotional states. Trends Neurosci. 16, 328-333 (1993).

19. Whalen, P.J. et al. Masked presentations of emotional facial expressions modulate amygdala activity without explicit knowledge. J. Neurosci. 18, 480-487 (1998).

20. Amaral, D.G. \& Price, J.L. Amygdalo-cortical projections in the monkey (Macaca fascicularis). J. Comp. Neurol. 230, 465-496 (1984).

21. Amaral, D.G., Behniea, H. \& Kelly, J.L. Topographic organization of projections from the amygdala to the visual cortex in the macaque monkey. Neuroscience 118 , 1099-1120 (2003)

22. Kilpatrick, L. \& Cahill, L. Amygdala modulation of parahippocampal and fronta regions during emotionally influenced memory storage. Neuroimage 20 2091-2099 (2003).

23. Meunier, M. \& Bachevalier, J. Comparison of emotional responses in monkeys with rhinal cortex or amygdala lesions. Emotion 2, 147-161 (2002).

24. Woermann, F.G., Barker, G.J., Birnie, K.D., Meencke, H.J. \& Duncan, J.S. Regional changes in hippocampal T2 relaxation and volume: a quantitative magnetic resonance imaging study of hippocampal sclerosis. J. Neurol. Neurosurg. Psychiatry 65 , 656-664 (1998).

25. Bartlett, P.A., Richardson, M.P. \& Duncan, J.S. Measurement of amygdala T2 relaxation time in temporal lobe epilepsy. J. Neurol. Neurosurg. Psychiatry 73, 753-755 (2002)

26. Hudson, L.P. et al. Amygdaloid sclerosis in temporal lobe epilepsy. Ann. Neurol. 33 , 622-631 (1993)
27. Wolf, H.K., Aliashkevich, A.F., Blumcke, I., Wiestler, O.D. \& Zentner, J. Neuronal loss and gliosis of the amygdaloid nucleus in temporal lobe epilepsy. A quantitative analysis of 70 surgical specimens. Acta Neuropathol. (Berl.) 93, 606-610 (1997).

28. Van Paesschen, W., Revesz, T., Duncan, J.S., King, M.D. \& Connelly, A. Quantitative neuropathology and quantitative magnetic resonance imaging of the hippocampus in temporal lobe epilepsy. Ann. Neurol. 42, 756-766 (1997).

29. Seeck, M. et al. Psychosocial functioning in chronic epilepsy: relation to hippocampal volume and histopathological findings. Epileptic Disord. 1, 179-185 (1999).

30. Adam, C. et al. Variability of presentation in medial temporal lobe epilepsy: a study of 30 operated cases. Acta Neurol. Scand. 94, 1-11 (1996).

31. Ashburner, J. \& Friston, K.J. Voxel-based morphometry-the methods. Neuroimage $11,805-821$ (2000)

32. Woermann, F.G., Free, S.L., Koepp, M.J., Ashburner, J. \& Duncan, J.S. Voxel-byvoxel comparison of automatically segmented cerebral gray matter-A raterindependent comparison of structural MRI in patients with epilepsy. Neuroimage 10, 373-384 (1999)

33. Wojciulik, E., Kanwisher, N. \& Driver, J. Covert visual attention modulates facespecific activity in the human fusiform gyrus: fMRI study. J. Neurophysiology 79, $1574-1578$ (1998).

34. Mishkin, M. Memory in monkeys severely impaired by combined but not by separate removal of amygdala and hippocampus. Nature 273, 297-298 (1978).

35. Catani, M., Jones, D.K., Donato, R. \& Ffytche, D.H. Occipito-temporal connections in the human brain. Brain 126, 2093-2107 (2003).

36. Baxter, M.G. \& Murray, E.A. The amygdala and reward. Nat. Rev. Neurosci. 3, 563-573 (2002).

37. Pessoa, L., McKenna, M., Gutierrez, E. \& Ungerleider, L.G. Neural processing of emotional faces requires attention. Proc. Natl. Acad. Sci. USA 99, 11458-11463 (2002).

38. Cahill, L. \& McGaugh, J.L. Mechanisms of emotional arousal and lasting declarative memory. Trends Neurosci. 21, 294-299 (1998).

39. Hamann, S.B. \& Adolphs, R. Normal recognition of emotional similarity between facial expressions following bilateral amygdala damage. Neuropsychologia 37, 1135-1141 (1999).

40. Adolphs, R., Baron-Cohen, S. \& Tranel, D. Impaired recognition of social emotions following amygdala damage. J. Cogn. Neurosci. 14, 1264-1274 (2002).

41. Meletti, S. et al. Impaired facial emotion recognition in early-onset right mesial temporal lobe epilepsy. Neurology 60, 426-431 (2003).

42. Siebert, M., Markowitsch, H.J. \& Bartel, P. Amygdala, affect and cognition: evidence from 10 patients with Urbach-Wiethe disease. Brain 126, 2627-2638 (2004).

43. Anderson, A.K. \& Phelps, E.A. Is the human amygdala critical for the subjective experience of emotion? Evidence of intact dispositional affect in patients with amygdala lesions. J. Cogn. Neurosci. 14, 709-720 (2002).

44. Cahill, L. et al. Amygdala activity at encoding correlated with long-term, free recall of emotional information. Proc. Natl. Acad. Sci. USA 93, 8016-8021 (1996).

45. Vuilleumier, P., George, N., Lister, V., Armony, J. \& Driver, J. Effects of perceived mutual gaze on face judgements and face recognition memory. Visual Cognition (in press).

46. Price, J.L. Comparative aspects of amygdala connectivity. Ann. N. Y. Acad. Sci. 985, 50-58 (2003).

47. Adolphs, R. Neural systems for recognizing emotion. Curr. Opin. Neurobiol. 12, 169-177 (2002)

48. Ekman, P. \& Friesen, W. Pictures of facial affect. (Consulting Psychologists Press, Palo Alto, USA, 1976).

49. Van Paesschen, W., Connelly, A., Johnson, C.L. \& Duncan, J.S. The amygdala and intractable temporal lobe epilepsy: a quantitative magnetic resonance imaging study. Neurology 47, 1021-1031 (1996)

50. Wieshmann, U.C. et al. Magnetic resonance imaging in epilepsy with a fast FLAIR sequence. J. Neurol. Neurosurg. Psychiatry 61, 357-361 (1996) 